\title{
TIME OPTIMAL CONTROL OF THE MOLECULAR WEIGHT IN A SEMI BATCH EMULSION POLYMERIZATION
}

\author{
R. Gesthuisen ${ }^{* 1}$, K. Dadhe *, S. Krämer* ${ }^{*}$ S. Engell ${ }^{*}$ \\ * Process Control Laboratory, \\ Department of Biochemical and Chemical Engineering, \\ University of Dortmund, D-44221 Dortmund, Germany, \\ Tel: +49/231/755-5127, Fax: +49/231/755-5129
}

\begin{abstract}
In this contribution a hierarchical approach to the control of the molecular weight in a semi-batch emulsion polymerization is derived, which ensures minimum batch time. Rather than performing a numerical optimization to determine an optimal trajectory for the process at any point in time, the existing boundary conditions are examined and used to determine the time optimal operation at each time step. This procedure provides the set points for the molar holdups of monomer and chain transfer agent (CTA) in the system. Either a decentralized PI controller or a nonlinear model predictive controller (NMPC) can be applied to track the calculated trajectory. As the set point calculation is a solution of a system of algebraic equations it can easily be performed on-line. It is shown that this concept can be used to produce multi modal molecular weight distributions and can also handle disturbances in the process, such as an unexpected nucleation of particles.
\end{abstract}

Keywords: optimization, emulsion polymerization, control, NMPC, molecular weight

\section{INTRODUCTION}

Semi-continuous emulsion polymerization (SEP) is one of the major processes in the production of polymer latices. Control of the molecular weight distribution is one major issue when running the process. Usually a seed, a pre-defined polymer dispersion with a known number of particles, particle size, and monomer concentration, is used to ensure well-known starting conditions. In order to produce a certain molecular weight distribution or a special co-polymer composition, control of the concentrations of the monomers and possibly of the chain transfer agent is necessary. Vicente et al. (2001) calculate an optimal trajectory by minimizing the inverse of the reaction rate to achieve an optimal process time. The equality constraints of this optimization problem are given by a rigorous dynamic model of the polymerization of styrene with butyl maleate $(\mathrm{BuM})$, which acts as a CTA. The resulting set points for the molar

\footnotetext{
1 Corresponding author

E-mail: r.gesthuisen@bci.uni-dortmund.de
}

holdups of styrene and BuM are tracked by controller based on model inversion. Similar control structures are reported by Gentric et al. (1999), Kravaris et al. (1989) or Seferlis and Kiparissides (2001). They use closed loop control concepts, i. e. trajectory tracking is applied. In each of these publications the numerical optimization is performed off-line as the computational effort is too demanding for an on-line application. Krämer et al. (2002) applied an on-line re-optimization to a semibath emulsion co-polymerization. In order to be able to re-calculate the optimal trajectory on-line, a simplified model for the co-polymer composition was used. Due to the simplifications, the model error may be problematic. In this paper, we propose a different strategy in which time optimality is guaranteed by operating the process at its constraints, which is an approach towards realizing an optimal operation and was proposed by Srinivasan et al. (2003). As a feedback controller has to be used to track the optimal set points, the proposed control scheme is of hierarchical structure.

The process investigated here is the emulsion polymer- 
ization of styrene using butyl maleate as a CTA (cf. (Vicente et al., 2001)). The goal is to produce a prespecified multi modal molecular weight distribution in minimum time.

\section{PROCESS AND MODEL DESCRIPTION}

The process investigated here is the seeded semi-batch emulsion polymerization of styrene (Vicente et al., 2001). In order to control the molecular weight, a chain transfer agent (BuM) is used. The reactor is precharged with a well defined seed and is operated at $60^{\circ} \mathrm{C}$. Temperature control is realized by a PI-controller. The process is simulated using a rigorous model that describes the volumes of water, polymer, the total volume in the reactor and the molar holdups of monomer and CTA, the concentration of initiator, the average number of radicals in the particle phase and the discretized molecular weight distribution. In contrast to many other publications we consider the jacket as as a plug flow reactor (PFR). This modification is necessary when pilot plants or industrial scale reactors are considered as the assumption of an ideally stirred jacket is not correct for reactors of larger volume. The resulting partial differential equation is approximated by the method of orthogonal collocation (the interested reader is referred to (Georgakis et al., 1977). It is assumed that the heat of reaction is only produced by the polymerization reaction, i. e. there is no contribution by the reaction of CTA. Furthermore, we assume that the heat transfer coefficient decreases with increasing conversion, i. e. with increasing solids content.

\section{HIERARCHICAL CONTROL APPROACH}

Figure 1 depicts the hierarchical control concept on which the control algorithm is based. It consists of four basic steps:

(1) Calculation of the average molecular weights which have to be produced in certain conversion intervals (computed off-line).

(2) Calculation of the set point of the molar monomer holdup in the reactor.

(3) Calculation of the ratio between the total molar holdups of monomer and of CTA.

(4) Calculation of the control actions by a controller (decentralized PI-controller or NMPC).

Each of these steps is described briefly in the subsequent paragraphs.

\section{Calculation of the average molecular weights}

For linear polymers formed in an ideal polymerization it is possible to describe an arbitrary MWD by the combination of ideal MWDs with certain average molecular weights (Vicente et al., 2001). Therefore, the free parameters in this optimization step are the average molecular weights of the different modes and the fractions of conversion for which these molecular weights are produced. Vicente et al. (2001) derive conditions for which the termination of radical chains in an emulsion polymerization with CTA is dominated by the chain transfer reaction and therefore by the ratio between the monomer and CTA concentration in the particle phase. For such a process the assumption that the instantaneous MWD is of Schulz-Zimm type holds. In this case the instantaneous average molecular weight is calculated by:

$$
\bar{M}_{n, i n s}=\frac{k_{p}[M]^{p}}{k_{t r, C T A}[C T A]^{p}} P_{M} .
$$

where $P_{M}$ denotes the molecular weight of the repeating unit. This equation holds for the following conditions:

$$
\frac{[C T A]^{p}}{[M]^{p}}>5.2 \times 10^{-6} \quad[C T A]^{p}>3.5 \times 10^{-6}\left[\frac{m o l}{l}\right] .
$$

The calculation is performed off-line, while all other calculations discussed in the sequel have to be performed on-line.

\section{Calculation of the set point of the molar monomer holdup in the reactor}

To determine the largest possible reaction rate two criteria have to be evaluated in order to find the highest possible reaction rate, i. e. the maximum admissible concentration of monomer in the particle phase. These two criteria are:

- A droplet phase has to be avoided.

- The heat generated by reaction must not exceed the maximum heat removal capability.

As a result of both calculations a maximum admissible number of moles is calculated, the minimum of the two is used as the set point of a lower level controller.

Avoiding a droplet phase When the maximum swelling of the polymer particles with monomer is reached, a droplet phase will form. As the reaction rate in the polymer particles depends solely on the amount of monomer in the particles, an additional droplet phase will not increase the reaction rate, but it will downgrade the controllability of the process as the droplets act as a reservoir and enable the direct influence on the concentrations in the particle phase. Thus, droplet phase is unnecessary and potentially dangerous and has to be avoided.

For the calculation of the maximum amount of monomer we will assume that the minimum amount of CTA has no strong influence on the phase distribution of the monomer, especially, when a safety margin for the maximum amount has been set. Thus, the necessary calculation is similar to the case of a homopolymerization. In an earlier paper, we calculated a condition for the nonexistence of a droplet phase (Krämer et al., 2001):

$$
V_{W} \geq\left(k^{d}-1\right) V_{M}^{w}
$$

If the maximum total amount of monomer allowed in 


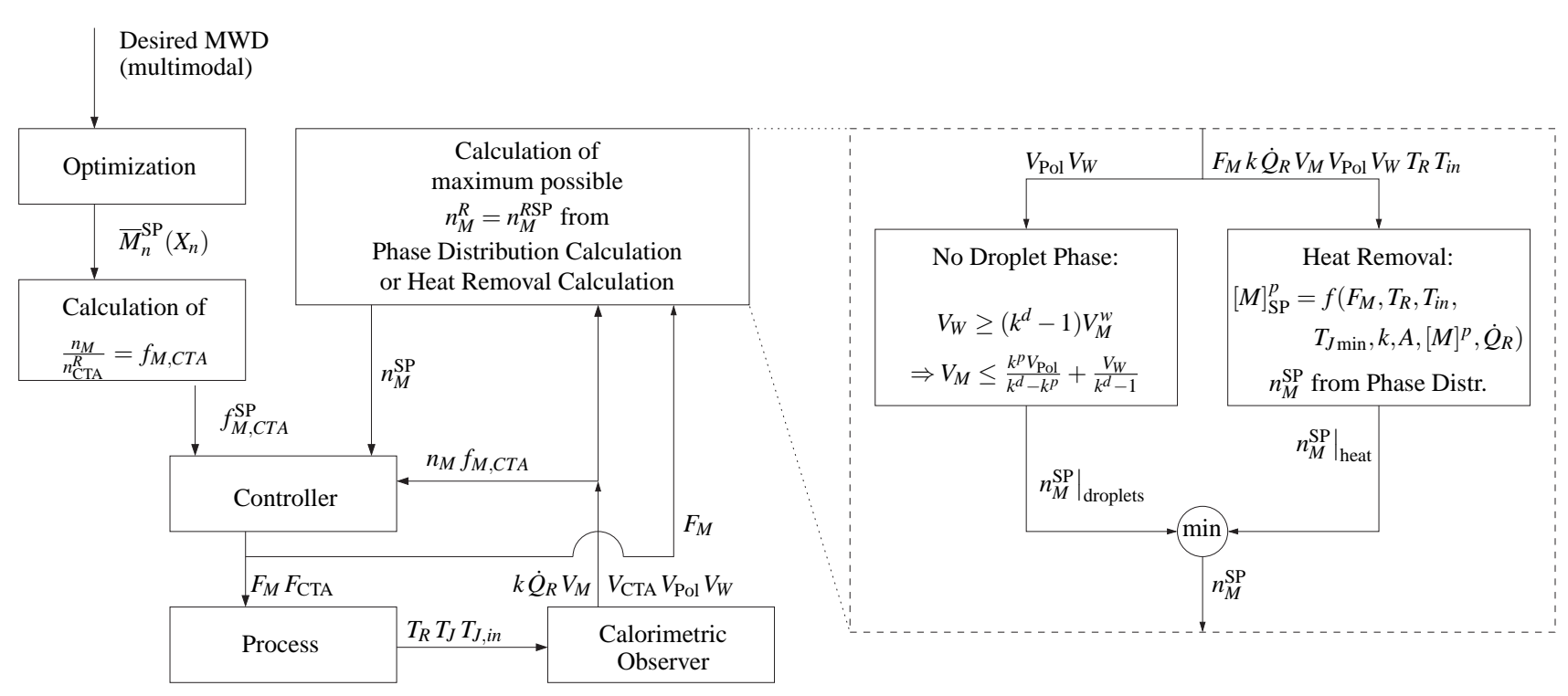

Fig. 1. Description and explanation of the hierarchical control concept applied

the system to avoid the formation of a droplet phase is to be calculated, the amount of water and the amount of polymer in the particles need to be known. Here, $V_{\mathrm{Pol}}$ and $V_{W}$ are estimated and calculated from the mass balance, respectively. Thus, using the following equations and inequality (3) as an additional equation, the maximum amount of monomer can be calculated:

$$
\begin{aligned}
k^{p} \frac{V_{M}^{w}}{V^{w}} & =\frac{V_{M}^{p}}{V^{p}} \\
V^{w} & =V_{M}^{w}+V_{W} \\
V^{p} & =V^{p}+V_{\mathrm{Pol}} \\
V_{M} & =V_{M}^{p}+V_{M}^{w} .
\end{aligned}
$$

The result of this system of equations is the following condition for the total molar holdup $n_{M}^{S P}$ :

$$
n_{M}^{S P}=\varphi\left(\frac{k^{p} V_{\mathrm{Pol}}}{k^{d}-k^{p}}+\frac{V_{W}}{k^{d}-1}\right) \bar{V}_{M}
$$

Here, $\varphi<1$ denotes a safety margin. With increasing conversion, e. g. with an increasing amount of polymer, the maximum amount of monomer will increase. On the other hand, the heat transfer coefficient will decrease significantly and will limit the heat removal and therefore the acceptable amount of free monomer.

Maximum Heat Removal To calculate the maximum heat removal, a number of internal states and the manipulated variables are needed. It has been shown that the inner states can be calculated by applying open loop observers as shown in (Vicente et al., 2001). The scope of this contribution is the derivation of the control concept. Thus, we assume that all required states in the following calculations are available.

Assume that a minimum jacket inlet temperature $T_{J, \text { min }}$ is given. Furthermore it is assumed that the process should be operated at isothermal conditions. Hence, from the stationary heat balance for the reactor and the assumption that the change of the heat capacity $C_{R}$ in time depends mainly on the feed to the reactor, the following expression for the maximum heat of reaction which can be removed by the jacket cooling is derived:

$$
\dot{Q}_{R, \max }=\dot{V}_{\text {in }} \rho_{\text {in }} c_{p, \text { in }}\left(T_{R}-T_{\text {in }}\right)-k A\left(\bar{T}_{J \min }-T_{R}\right) . .
$$

Here, $\bar{T}_{J \text { min }}$ the specified minimum average temperature allowed in the jacket, i.e. in case of a CSTR-jacket it is the specified outlet temperature and in case of a PFRjacket it is the mean temperature over the length of the pipe. This temperature is obviously depended on the minimum jacket inlet temperature.

The heat of reaction currently produced in the reactor is defined by:

$$
\dot{Q}_{R}=k_{p}[M]^{p} \frac{\bar{n} N_{T}}{N_{A}}(-\Delta H) .
$$

From the ratio between the currently produced heat of reaction $\dot{Q}_{R}(10)$ and the maximum removable heat of reaction $\dot{Q}_{R, \max }(9)$ an equation for the maximum allowed concentration of monomer in the particle phase can be derived:

$$
[M]_{\max }^{p}=\left(\dot{V}_{i n} \rho c_{p}\left(T_{R}-T_{i n}\right)-k A\left(\bar{T}_{J \min }-T_{R}\right)\right) \frac{[M]^{p}}{\dot{Q}_{R}}
$$

By adjusting $T_{J \min }$ a safety margin can be defined. The desired set-point $n_{M}^{\text {SP }}$ can now be calculated from the inverse of the phase distribution calculation. Alternatively and less complex it can be assumed that no droplet phase exists and the solubility of the monomer in water is negligible:

$$
n_{M}^{\mathrm{SP}}=[M]_{\max }^{p} \frac{V_{P o l}}{1-[M]_{\max }^{p} \bar{V}_{M}} .
$$

This simplification is valid as the non-existence of a 
droplet phase is checked by the foregoing criterion.

\section{Calculation of the total molar holdup ratio}

The off-line calculation of step 1 gives the ratio $f_{M . C T A}^{p}$ between monomer and CTA in the polymer particle phase. As the controlled variables are the total amounts of the reactants, the desired ratio of these values must be determined. Hence, the phase distribution algorithm (Arzamendi et al., 1998) has to be reformulated and extended by the following equation:

$$
V_{C T A}=\frac{\bar{V}_{C T A} V_{M}}{\bar{V}_{M} f_{M, C T A}^{p}} \frac{1+\frac{V^{w}}{V^{p} k_{C T A}^{p}}+\frac{V^{d} k_{C T A}^{d}}{V^{p} k_{C T A}^{p}}}{1+\frac{V^{w}}{V^{p} * k_{M}^{p}}+\frac{V^{d} k_{M}^{d}}{V^{p} k_{M}^{p}}} .
$$

By introducing this equation the amount of CTA in the particle phase is fixed, but the total amount of CTA in the reactor becomes a free variable. From the solution of the modified phase distribution algorithm the desired ratio is given by

$$
f_{M, C T A}=\frac{V_{M} \bar{V}_{C T A}}{V_{C T A} \bar{V}_{M}}
$$

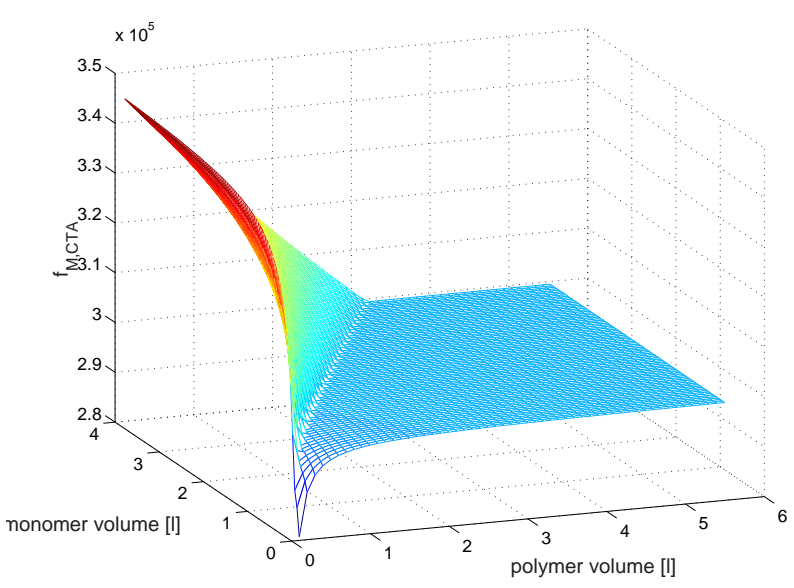

Fig. 2. Dependency of $f_{M, C T A}$ on the polymer volume and the monomer volume

Figure 2 shows the dependency of the ratio $f_{M, C T A}$ on the polymer and monomer volume. The desired ratio in the polymer is $f_{M, C T A}^{p}=3 \times 10^{5}$. It follows that for a wide range of polymer and monomer volumes the two ratios (particle and reactor) are nearly identical. If a droplet phase exists, the necessary ratio of the molar holdup of monomer and CTA in the reactor differs significantly from the desired ratio in the particle phase.

$\bar{V}_{C T A}$ and $\bar{V}_{M}$ denotes the molar volumes of the CTA and the monomer, respectively. The set point of the total molar holdup reads as:

$$
n_{C T A}^{S P}=\frac{n_{M}^{S P}}{f_{M, C T A}} .
$$

In this manner, the process is always driven under time optimal conditions as it is performed at its upper constraints (Srinivasan et al., 2003). Finally, controllers have to be designed that track the given set point trajectory.

\section{Controller design}

We investigated two types of controllers, simple decentralized PI-controllers for each of the molar holdups and an NMPC. The controlled variables are the total amounts of monomer and CTA in the reactor and the manipulated variables are defined by the molar flow rates of the to reactants.

NMPC-design The dynamic model used in the NMPC scheme is based on the rigorous model described in section 2 . The state variables are:

- total reactor volume

- overall amount of monomer and CTA

- volume of water

- concentration of initator

- polymer volume

- concentration of radicals in the water phase

- average number of radicals per particle.

The NMPC is realized in a sequential approach using the SQP-routine fmincon.m provided by MATLAB. In order to make the computation more effective, the phase distribution equations were linearized around the initial states of the NMPC in each step what enables the formulation of an analytic solution. As a linear controller is used to keep the reactor temperature at the desired set point, it is unnecessary to take the heat balance equations for the reactor and the jacket into account for the NMPC. Different prediction and control horizon lengths were checked and tuning of the weighting matrices was performed by simulation studies. The cost function is chosen as:

$$
J=\left|n^{S P}-n\right|_{Q_{x}}^{2}+|\Delta u|_{Q_{\Delta u}}^{2} .
$$

The first term assesses the deviation from the desired set points of the molar holdups of monomer and CTA and the second term weights the changes of the manipulated variables to avoid too fast control actions. The upper and lower bounds for the flow rates are given by the equipment of the pilot plant.

Decentralized PI-controller design In general, emulsion polymerization processes are highly nonlinear in nature. Due to the avoidance of a droplet phase in the operation of the process and due to the fact, that monomer and CTA do not interact, the nonlinearities are not strong. Therefore, it is also possible to design linear decentralized controllers to track the set points of the molar holdups of monomer and CTA. The gains of the controllers were adjusted by simulation studies.

The proposed hierarchical control approach can also be applied to controlling the composition in a copolymerization. In this case the coupling between the different monomers is strong and the nonlinearities are significant. It is expected that in this situation an NMPC outperforms a linear MIMO-controller. 

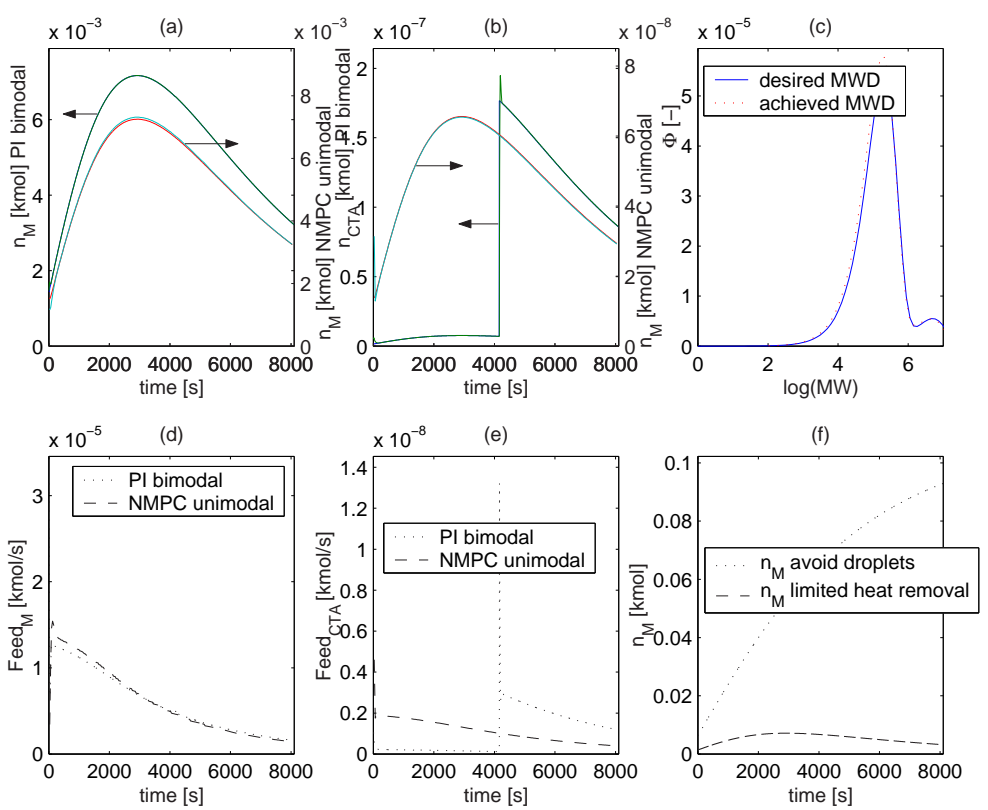

Fig. 3. Performance of the NMPC and the decentralized PI-controller scheme - PI controlled process: production of a bimodal MWD - NMPC controlled process: production of a uni-modal MWD - (a) Tracking of the molar holdup of monomer $n_{M}$, (b) Tracking of the molar holdup of CTA $n_{C T A}$, (c) comparison between desired and achieved MWD for PI-controlled process, (d) molar feed monomer Feed $_{M}$, (e) molar feed CTA Feed $_{C T A}$, (f) development of the constraints over batch time

\section{SIMULATION STUDIES}

The simulation results are obtained for a polymerization in a 101 stainless steel reactor with geometric parameters taken from a pilot plant at the Process Control Laboratory at the University of Dortmund. Approximately 7\% of the total polymer to be produced is pre-charged as a seed. The production of uni-modal as well as multimodal molecular weight distributions was investigated. Figure 3(c) shows the simulation results for the production of a bi-modal molecular weight distribution using decentralized PI-controllers. It follows that the control strategy is able to produce the desired MWD. Figures 3(a-b) and (d-e) depict the set point tracking and the manipulated variables, e.g. the molar feed rates of monomer and CTA, for either an NMPC- (uni-modal) and PIcontrolled (bi-modal) process. The NMPC was investigated as it is most often stated in the open literature that nonlinear control is required for this type of process. The simulation results in this contribution clearly demonstrate that this is not necessary. Figures 3 (a-b) demonstrate that both approaches are able to track the desired set points very quickly. Certainly, there is a difference in the performance between the NMPC-scheme and the decentralized PI-controllers as the NMPC is operated with a sample time of 30 seconds whereas the time discrete PI controllers are performed with a sample time of 3 seconds. The different sampling intervals result from the different computational effort to calculate the manipulated variables.

Due to the pre-charged seed, the upper constraints of the process are completely determined by the possible heat removal, see figure 3 (f). The set point of the molar holdup in the reactor increases for the first third of the process time. The reason for this behaviour is the increase of the polymer volume. As the reaction rate depends only on the concentration of the monomer in the polymer phase, it is possible to increase the total amount of monomer in the reactor without increasing the reaction rate. With increasing conversion/process time the decreasing heat removal capacity becomes more and more important and leads to a decrease of the allowed total amount of monomer in the reactor. Hence, the simulation results show that the hierarchical control approach is a possible solution to time optimal operation of the emulsion polymerization process at hand.

Figure 4 depicts the trajectory of the desired ratio of monomer and CTA and the output of the controlled process versus the total conversion. The control algorithm is able to track the desired ratio quickly. It has to be considered that in the beginning of the process the reaction rate is high and the conversion increases fast. It takes the PI-controllers less than 260 seconds to bring the process to a tolerance band of $\pm 1 \%$ of the set point.

Figure 5 depicts the temperatures in the reactor and the jacket inlet for a disturbed process. It is assumed that an additional nucleation occurs at $t=5000$ seconds. If an off-line calculated trajectory for the amounts of monomer and CTA is used, it is not possible to keep the temperature of the reactor at the desired value as the reaction rate increases significantly due to the larger number of reaction loci and the too large amount of monomer in the system. The proposed hierarchical control approach performs well and adjusts the trajectory of the monomer holdup to the new reaction conditions. The set point of the total amount of monomer is therefore an 


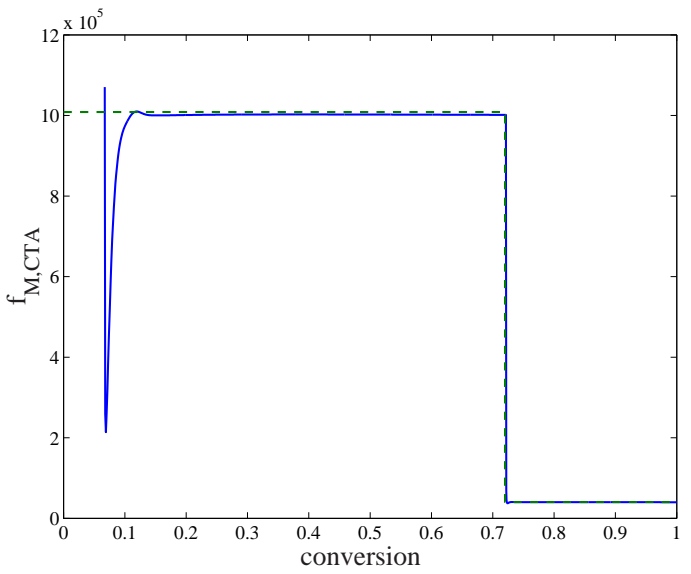

Fig. 4. Performance of the decentralized PI-controller scheme - tracking of the desired ratio $f_{M, C T A}$ (dashed: set point, solid: achieved ratio)

additional degree of freedom in the process which cannot be used if an off-line calculated trajectory is applied.

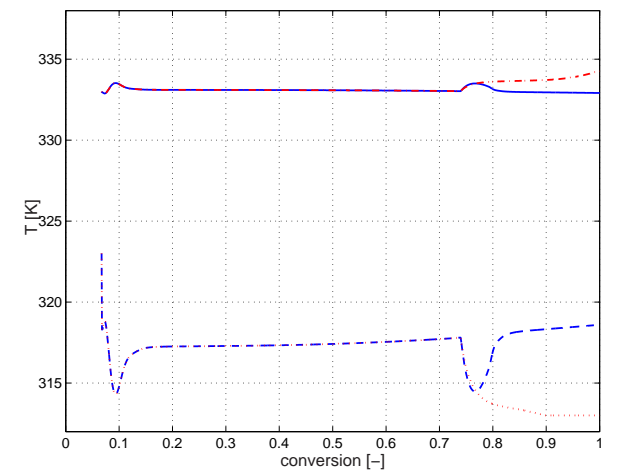

Fig. 5. Temperature control: Comparison between the performance of the proposed approach and an offline calculated optimal trajectory if an additional nucleation step occurs. solid: $T_{R}$ proposed, dashed: $T_{J, \text { in }}$ proposed, dash-dot: $T_{R}$ off-line, dotted: $T_{J, i n}$ off-line

\section{CONCLUSIONS}

A new hierarchical control approach for a time optimal production of a polymer with a prescribed molecular weight distribution in an emulsion polymerization using CTA has been developed. This approach avoids an offline optimization to calculate trajectories for monomer and CTA. Instead, the constraints of the process have been investigated and are used to calculate at each point in time set points for the molar holdups of monomer and CTA. The desired molecular weight distribution is approximated by a combination of ideal distributions. The average molecular weights of these ideal distribution are produced for a certain fraction of conversion. The maximum possible amount of monomer is calculated by the avoidance of a droplet phase or the maximum heat removal capacity, respectively. Appropriate conditions were derived. Applying a modified phase distribution algorithm, the total amount of CTA can be calculated and finally it can be used as set point in a nonlinear model predictive controller or a decentralized PIcontroller. It was shown by simulation that the derived approach performs well and is able to produce arbitrary multi modal molecular weight distributions. Another demonstrated feature of the proposed approach is the possibility to reject disturbances in the process, which cannot be achieved with off-line calculated trajectories.

\section{ACKNOWLEDGEMENTS}

Parts of this work were founded by the German Research Foundation (DFG-Deutsche Forschungsgemeinschaft, DFG-EN 152/31-1). The authors gratefully acknowledge this financial support.

\section{REFERENCES}

Arzamendi, Gurutze, Claudia Sayer, Nuria Zoco and José M. Asua (1998). Modeling of MWD in emulsion polymerization: Partial distinction approach. Polymer Reaction Engineering 6(3-4), 193-223.

Gentric, C., F. Pla, M. A. Latifi and J. P. Corriou (1999). Optimization and nonlinear control of a batch emulsion polymerization reactor. Chemical Engineering Journal 75, 31-46.

Georgakis, C., R. Aris and N. R. Amundson (1977). Studies in the control of tubular reactors. Chemical Engineering Science 32, 1359-1369.

Krämer, Stefan, Ralf Gesthuisen and Sebastian Engell (2002). Re-optimization based control of copolymer quality in semi continuous emulsion polymerization. In: Proceedings of the American Control Conference (R. Russell Rhinehart, Ed.). American Automatic Control Council. Anchorage, USA. pp. 3870-3875.

Krämer, Stefan, Ralf Gesthuisen, Michael Wieland and Sebastian Engell (2001). An alternative model formulation for emulsion polymerization processes. In: Conference Proceedings of the European Congress of Chemical Engineering (VDI, Ed.). VDI-GVC. VDI-GVC. Frankfurt, Germany.

Kravaris, C., R. A. Wright and J. F. Carrier (1989). Nonlinear controllers for trajectory tracking in batch processes. Computers and Chemical Engineering 13(1/2), 73-82.

Seferlis, P. and C. Kiparissides (2001). Optimizing control of molecular weight properties for batch freeradical polymerization reactors. IFAC pp. 251-256.

Srinivasan, B., S. Palanki and D. Bonvin (2003). Dynamic optimization of batch processes. i. characterization of the nominal solution. Computers and Chemical Engineering 27, 1-26.

Vicente, Matias, Selwa Ben Amor, Luius M. Gugliotta, Jose R. Leiza and Jose M. Asua (2001). Control of molecular weight distribution in emulsion polymerization using on-line reaction calorimetry. Industrial and Engineering Chemistry Research 40(1), 218-227. 